\title{
Effect of Toki-Shakuyaku-San on Regional Cerebral Blood Flow in Patients with Mild Cognitive Impairment and Alzheimer's Disease
}

\author{
Teruyuki Matsuoka, ${ }^{1}$ Jin Narumoto, ${ }^{1}$ Keisuke Shibata, ${ }^{1}$ Aiko Okamura, ${ }^{1}$ Shogo Taniguchi, ${ }^{1}$ \\ Yurinosuke Kitabayashi, ${ }^{2}$ and Kenji Fukui ${ }^{1}$ \\ ${ }^{1}$ Department of Psychiatry, Graduate School of Medical Science, Kyoto Prefectural University of Medicine, 465 Kajii-cho, \\ Kawaramachi-Hirokoji, Kamigyo-ku, Kyoto 602-8566, Japan \\ ${ }^{2}$ Department of Psychiatry, Gojouyama Hospital, 4-6-3 Rokujo-Nishi, Nara 630-8044, Japan
}

Correspondence should be addressed to Teruyuki Matsuoka, tmms2004@koto.kpu-m.ac.jp

Received 12 October 2011; Accepted 18 November 2011

Academic Editor: Paul Siu-Po Ip

Copyright (C) 2012 Teruyuki Matsuoka et al. This is an open access article distributed under the Creative Commons Attribution License, which permits unrestricted use, distribution, and reproduction in any medium, provided the original work is properly cited.

The aim of this study was to examine the effect of toki-shakuyaku-san (TSS) on mild cognitive impairment (MCI) and Alzheimer's disease $(\mathrm{AD})$ using single-photon emission computed tomography (SPECT). All subjects were administered TSS (7.5 g/day) for eight weeks. SPECT and evaluations using the Mini Mental State Examination (MMSE), Neuropsychiatric Inventory, and Physical Self-Maintenance Scale were performed before and after treatment with TSS. Three patients with MCI and five patients with AD completed the study. No adverse events occurred during the study period. After treatment with TSS, regional cerebral blood flow $(\mathrm{rCBF})$ in the posterior cingulate was significantly higher than that before treatment. No brain region showed a significant decrease in rCBF. TSS treatment also tended to improve the score for orientation to place on the MMSE. These results suggest that TSS could be useful for treatment of MCI and AD.

\section{Introduction}

Cholinesterase inhibitors (ChEIs) and memantine, an Nmethyl-D-aspartate antagonist, are commonly used in treatment of Alzheimer's disease (AD). These drugs slow the progression of the disease and improve cognition, function, and behavior impairment but often have to be discontinued because of adverse events [1,2]. There are no approved drugs for treatment of mild cognitive impairment (MCI). A systematic review showed that ChEIs were ineffective in preventing progression of MCI to AD or improving cognitive functions and led to adverse events [3].

There has been a recent increase in the use of traditional herbal medicine for treatment of dementia. Yokukan-san has been shown to improve behavioral and psychological symptoms of dementia (BPSD) $[4,5]$, hachimi-jio-gan improved cognitive function and activity of daily living (ADL) in $\mathrm{AD}$ [6], and choto-san was effective for patients with vascular dementia [7, 8]. Moreover, combination treatment with kami-untan-to and donepezil improved cognitive function and increased regional cerebral blood flow $(\mathrm{rCBF})$ in the bilateral frontal lobes [9].

Toki-shakuyaku-san (TSS) is mainly used for gynecologic disorders but has also been used for treatment of cognitive impairment based on accumulated evidence of its neuroactive and neuroprotective effects. TSS activates cholinergic $[10-14]$ and monoaminergic $[12,13,15]$ neurons and has a protective effect on amyloid $\beta[16,17]$, an antioxidant effect $[18,19]$, and an antiapoptosis action [12]. Several clinical studies have also suggested that TSS improves cognitive impairment in dementia, MCI, and poststroke patients [2022]. Brain imaging may be useful to examine the effects of TSS. Therefore, the aim of this study was to identify the effects of TSS in patients with MCI and AD using singlephoton emission computed tomography (SPECT). 


\section{Methods}

2.1. Subjects. The subjects were 13 patients treated at the Center for Diagnosis of Dementia at the Kyoto Prefectural University of Medicine. Four patients were diagnosed with MCI and 9 with AD, based on Petersen et al. [23] and the National Institute of Neurological and Communicative Disease and Stroke-Alzheimer's Disease and Related Disorders Association (NINCDS-ADRDA) criteria for probable AD [24]. We excluded patients with a significant history of psychiatric or neurological disorders (other than MCI and AD), including stroke, head injury, epilepsy, psychiatric disorders, alcohol abuse, or a serious medical condition. Participants had not been prescribed ChEIs or memantine since our center examines patients who have not been diagnosed with dementia. This study was approved by the Ethics Committee of the Kyoto Prefectural University of Medicine. Informed consent was obtained from all of the patients.

2.2. Study Protocol. Subjects were treated with TSS given as a daily dose of $7.5 \mathrm{~g}$ of powder for eight weeks. During this period, new medications were not introduced. TSS is registered in the Pharmacopoeia of Japan as Kampo Medicine TJ-23. The TSS used in the study was provided by Tsumura (Tokyo, Japan) and was prepared from the extract of a mixture of dried plants: $4.0 \mathrm{~g}$ Paeoniae radix, $4.0 \mathrm{~g}$ Atractylodis lanceae rhizoma, $4.0 \mathrm{~g}$ Alismatis rhizoma, $4.0 \mathrm{~g}$ Hoelen, 3.0 g Cnidii rhizome, and $3.0 \mathrm{~g}$ Angelicae radix. All subjects underwent magnetic resonance imaging (MRI) or computed tomography (CT) before treatment. SPECT was performed before and after treatment.

Cognitive impairment was evaluated using the Mini Mental State Examination (MMSE) [25] before and after the study period. The MMSE has 11 subscales including orientation to time, orientation to place, registration, attention, recall, naming, repetition, auditory comprehension/command, reading comprehension, sentence construction, and constructional praxis. BPSD were evaluated before and after the study period using the Neuropsychiatric Inventory (NPI) [26]. The NPI is a caregiver-based clinical instrument that evaluates 10 domains of neuropsychiatric symptoms in dementia: delusions, hallucinations, agitation, depression, anxiety, euphoria, apathy, disinhibition, irritability, and aberrant motor behavior. The frequency score ranges from 0 to 4 points, and the severity score ranges from 0 to 3 points. The NPI score for each subscale is created by multiplying the frequency and severity scores, with a maximum score of 12. Therefore, the NPI total score ranges from 0 to 120 . Higher scores denote a greater severity of a symptom. ADL before and after the study period were evaluated using the Physical Self-Maintenance Scale (PSMS) [27], which consists of 6 items related to physical activities: toileting, feeding, dressing, grooming, ambulating, and bathing. A lower total score indicates greater impairment of ADL.

2.3. Image Acquisition and Analysis. Brain perfusion SPECT was performed by intravenous injection of $185 \mathrm{MBq}$ of $\mathrm{N}$-isopropyl-p-[ $\left.{ }^{123} \mathrm{I}\right]$ iodoamphetamine (I-123-IMP) (Nihon
Mediphysics, Hyogo, Japan) in subjects seated at rest with their eyes open. SPECT imaging commenced 22 min after the injection and continued for $16 \mathrm{~min}$. A triple-head gamma camera (Prism Irix, Picker International, Cleveland, $\mathrm{OH}, \mathrm{USA}$ ) and a low-energy, high-resolution, and parallel collimator were used. Projection data from each camera were obtained in a $128 \times 128$ format for 40 angles of 1201 at $8 \mathrm{~s}$ per angle (voxel size: $2 \times 2 \times 2 \mathrm{~mm}$ ).

Image analysis was performed using Statistical Parametric Mapping (SPM) 8 (Wellcome Department of Cognitive Neurology, University College, London, UK) in Matlab 7.5 (Mathworks Inv., Sherborn, MA, USA). After confirmation of no significant artifacts due to atrophy using MRI or CT scans, all SPECT images were anatomically normalized using the I-123-IMP template (Fujifilm RI Pharma, Tokyo, Japan) matched to the Montreal Neurological Institute (MNI) template. The normalized images were smoothed using a 12-mm full-width half-maximum (FWHM) isotropic Gaussian kernel. To examine regional differences, the images were scaled to a mean global cerebral blood flow of $50 \mathrm{~mL} / 100 \mathrm{~g} / \mathrm{min}$.

2.4. Statistical Analysis. A Wilcoxon signed rank test was used to analyze the changes in MMSE, NPI, and PSMS scores. Data were analyzed using SPSS $12.0 \mathrm{~J}$ for Windows (SPSS Inc., Chicago, IL, USA). $P<0.05$ was considered statistically significant. A paired $t$-test was performed to determine whether TSS affected rCBF in patients with MCI and $\mathrm{AD}$. The $X, Y$, and $Z$ coordinates provided by SPM approximate the MNI brain space. The statistical thresholds were set to a family-wise error (FWE)-corrected $P$ value of 0.05 at the voxel level.

\section{Results}

3.1. Subject Characteristics. Eight of the 13 subjects completed the study. Five were discontinued because of poor compliance $(n=3)$, a move to another area $(n=1)$, and refusal to continue $(n=1)$. None of the subjects had adverse events. The characteristics of the 8 subjects who completed the study are shown in Table 1. Six had not been prescribed with a psychoactive drug, 1 had taken risperidone ( $1 \mathrm{mg} /$ day), and 1 had taken rilmazafone $(0.5 \mathrm{mg} /$ day $)$ before the start of the study. Both subjects continued to take these drugs during the study.

3.2. Changes in MMSE, NPI, and PSMS Scores. Changes in MMSE, NPI, and PSMS scores are shown in Table 2. At baseline, cognitive impairment and BPSD were mild, and ADL was high. Scores for the MMSE, NPI total, and PSMS did not change significantly after TSS treatment. Among the MMSE subscales, the score for orientation to place showed a tendency to improve $(P=0.025)$, but the change was not significant using a Bonferroni correction $(P=0.025>$ $0.05 / 11$ ) (Table 3).

3.3. Paired $t$-Test Using SPM. The paired $t$-test showed a significant increase in $\mathrm{rCBF}$ in the posterior cingulate after TSS treatment compared to before treatment (Table 4). 
TABle 1: Clinical characteristics of subjects who completed the study.

\begin{tabular}{lc}
\hline Item & Value \\
\hline Sex, M/F & $3 / 5$ \\
Handedness, R/L & $8 / 0$ \\
Age, y.o. & $77.8 \pm 4.9$ \\
Diagnosis, MCI/AD & $3 / 5$ \\
Age at onset, y.o. & $76.3 \pm 4.3$ \\
Duration of illness, years & $1.6 \pm 1.6$ \\
Education, years & $12.0 \pm 1.7$ \\
\hline
\end{tabular}

AD: Alzheimer's disease; F: female; L: left; M: male; MCI: mild cognitive impairment; R: right; SD: standard deviation; y.o.: years old.

Values are shown as a number ratio or as the mean \pm SD.

TABle 2: Changes in MMSE, NPI and PSMS scores from before to after TSS treatment for 8 weeks.

\begin{tabular}{lccc}
\hline & $\begin{array}{c}\text { Baseline } \\
\text { mean } \pm \text { SD }\end{array}$ & $\begin{array}{c}8 \text { weeks } \\
\text { mean } \pm \text { SD }\end{array}$ & $P$ value \\
\hline MMSE & $23.4 \pm 3.6$ & $23.9 \pm 3.8$ & 0.279 \\
NPI total score & $5.5 \pm 5.9$ & $3.9 \pm 4.6$ & 0.180 \\
PSMS & $5.9 \pm 0.4$ & $5.4 \pm 1.4$ & 0.414
\end{tabular}

MMSE: Mini Mental State Examination; NPI: Neuropsychiatric Inventory; PSMS: Physical Self-Maintenance Scale; SD: standard deviation.

TABLE 3: Changes in MMSE subscale scores from before to after TSS treatment for 8 weeks.

\begin{tabular}{lccc}
\hline & $\begin{array}{c}\text { Baseline } \\
\text { mean } \pm \text { SD }\end{array}$ & $\begin{array}{c}8 \text { weeks } \\
\text { mean } \pm \text { SD }\end{array}$ & $P$ value \\
\hline Orientation to time & $3.5 \pm 1.6$ & $3.1 \pm 2.1$ & 0.414 \\
Orientation to place & $3.9 \pm 0.4$ & $4.5 \pm 0.5$ & 0.025 \\
Registration & $3.0 \pm 0.0$ & $3.0 \pm 0.0$ & 1.000 \\
Attention & $3.5 \pm 1.6$ & $3.4 \pm 1.6$ & 0.655 \\
Recall & $1.0 \pm 1.2$ & $1.0 \pm 0.9$ & 0.891 \\
Naming & $2.0 \pm 0.0$ & $2.0 \pm 0.0$ & 1.000 \\
Repetition & $0.9 \pm 0.4$ & $1.0 \pm 0.0$ & 0.317 \\
Auditory & $2.8 \pm 0.5$ & $3.0 \pm 0.0$ & 0.157 \\
comprehension/command & & & \\
Reading comprehension & $1.0 \pm 0.0$ & $1.0 \pm 0.0$ & 1.000 \\
Sentence construction & $1.0 \pm 0.0$ & $1.0 \pm 0.0$ & 1.000 \\
Constructional praxis & $0.9 \pm 0.4$ & $0.9 \pm 0.4$ & 1.000 \\
\hline
\end{tabular}

MMSE: Mini Mental State Examination; SD: standard deviation.

When the statistical thresholds were set to an uncorrected $P$ value of 0.001 at the voxel level and a corrected $P$ value of 0.05 at the cluster level, the posterior cingulate was the only brain region that showed a significant increase in $\mathrm{rCBF}$ (Figure 1). No brain region showed a significant decrease in $\mathrm{rCBF}$.

\section{Discussion}

In this study, $\mathrm{rCBF}$ in the posterior cingulate was significantly increased after eight weeks of treatment with TSS. The MMSE, NPI total, and PSMS scores did not worsen and adverse events did not occur during the treatment. Moreover,
TSS treatment tended to improve the score for orientation to place on the MMSE.

Some clinical studies have demonstrated improvement in cognitive impairment by TSS in dementia and poststroke patients. Inanaga et al. reported improvement in orientation to time and place, spontaneous activity, emotional lability, and motivation in 80 dementia patients (40 with vascular dementia, 38 with $\mathrm{AD}$, and 2 with mixed dementia) after 12 weeks of TSS treatment [20], while Goto et al. demonstrated that TSS is effective in suppressing impairment of visuospatial perception and the lower limbs in poststroke patients [22]. In our subjects, orientation to place tended to improve while other cognitive impairments and BPSD were unchanged. These findings are partly consistent with previous studies and suggest that TSS might be particularly effective for improvement of spatial perception.

TSS treatment in this study was associated with a change in $\mathrm{rCBF}$ in the posterior cingulate. This brain region plays an important role in many cognitive functions, including visuospatial orientation, topokinesis, navigation of the body in space, self reflection, autobiographical memory, and assessment of objects in space in terms of first-person orientation [28]. Connections between the posterior cingulate and the parahippocampus are likely to play a major role in memory-related functions [29], and these connections may be disturbed in $\mathrm{MCI}$ and $\mathrm{AD}[30,31]$. Moreover, hypofunction in the posterior cingulate is associated with visuoperceptual deficits in $\mathrm{MCI}$ and $\mathrm{AD}$ [32], as well as disorientation to time and place in $\mathrm{AD}$ [33]. In this study, TSS increased $\mathrm{rCBF}$ in the posterior cingulate and improved orientation to place based on the MMSE. Therefore, TSS might be useful for treatment of cognitive impairment associated with the posterior cingulate.

Previous studies have shown that donepezil increases or maintains rCBF, mainly in the frontal lobes [34-37]. In contrast, in this study, TSS increased rCBF in the posterior cingulate. In addition to the cholinergic effect, TSS also has monoaminergic, antiamyloid, antioxidant and antiapoptosis effects [10-19]. Collectively, these results suggest that TSS has different effects on cognitive impairment compared to those of donepezil. Therefore, combining TSS with donepezil might have an additional benefit in treatment of MCI and AD.

The study has several limitations, since it was performed with an open label design with no control group, the observation period was short, and the sample size was small. These limitations may reduce the strength of the results, but the findings were partly consistent with previous studies. Moreover, to our knowledge, this is the first study to demonstrate an effect of TSS on rCBF in patients with MCI and AD.

\section{Conclusions}

Treatment with TSS significantly increased rCBF in the posterior cingulate and tended to improve orientation to place in $\mathrm{MCI}$ and $\mathrm{AD}$ patients. Therefore, TSS might be useful for treatment of MCI and AD. Moreover, since the effects of TSS on rCBF may differ from those of donepezil, combination 
TABLE 4: Results of paired $t$-tests.

\begin{tabular}{lccccccc}
\hline Brain area & \multicolumn{2}{c}{ MNI coordinates at the center of the cluster } & $\begin{array}{c}Z \text { value at the local } \\
\text { maximum }\end{array}$ & $\begin{array}{c}\text { Voxel } P \text { value } \\
\text { (corrected) }\end{array}$ & $\begin{array}{c}\text { Cluster } \\
\text { size }\end{array}$ & $\begin{array}{c}\text { Cluster } P \text { value } \\
\text { (corrected) }\end{array}$ \\
\hline \multirow{2}{*}{ Posterior cingulate } & $X$ & $Y$ & $Z$ & 5.66 & 0.002 & 6 & $<0.001$ \\
& 2 & -32 & 40 & 44 & 5.08 & 0.039 & 1 \\
\hline
\end{tabular}
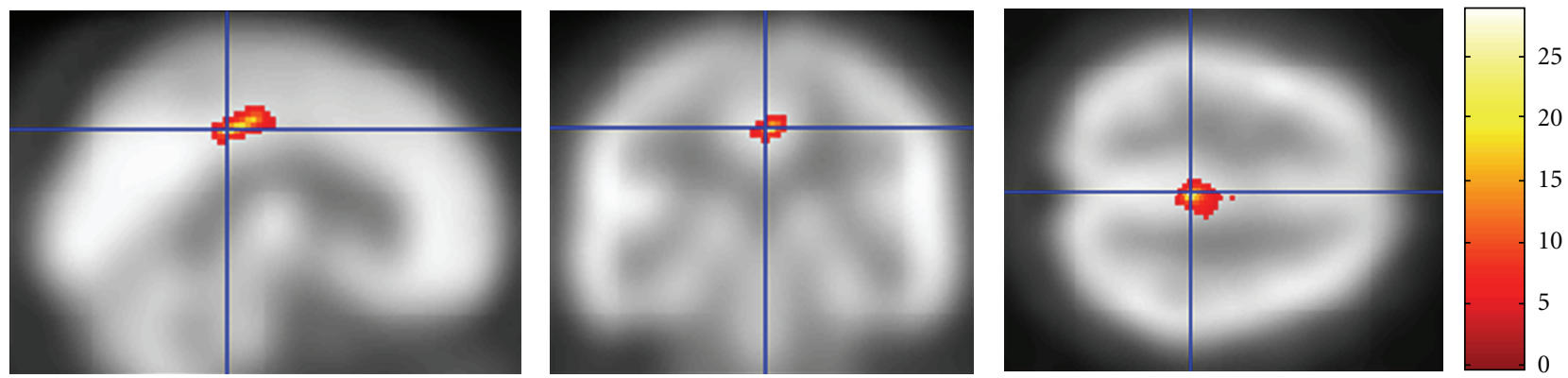

FIgURE 1: Regions showing a significant increase in $\mathrm{rCBF}$ after TSS treatment. The statistical thresholds were set to an uncorrected $P$ value of 0.001 at the voxel level and to a corrected $P$ value of 0.05 at the cluster level.

therapy of TSS and donepezil might be particularly effective. A study in a large number of MCI and AD patients is needed to confirm the effects of TSS.

\section{Conflict of Interests}

The authors declare that there is no conflict of interests.

\section{Acknowledgments}

This study was supported by a Grant-in-Aid for Young Scientists (B) (19790829) from the Ministry of Education, Culture, Sports, Science and Technology (MEXT) (to Dr. Y. Kitabayashi) and by the research fund of the Institute of Kampo Medicine.

\section{References}

[1] R. A. Hansen, G. Gartlehner, A. P. Webb, L. C. Morgan, C. G. Moore, and D. E. Jonas, "Efficacy and safety of donepezil, galantamine, and rivastigmine for the treatment of Alzheimer's disease: a systematic review and meta-analysis," Clinical Interventions in Aging, vol. 3, no. 2, pp. 211-225, 2008.

[2] S. J. Thomas and G. T. Grossberg, "Memantine: a review of studies into its safety and efficacy in treating Alzheimer's disease and other dementias," Clinical Interventions in Aging, vol. 4, pp. 367-377, 2009.

[3] R. Raschetti, E. Albanese, N. Vanacore, and M. Maggini, "Cholinesterase inhibitors in mild cognitive impairment: a systematic review of randomised trials," PLoS Medicine, vol. 4, no. 11, article 338, 2007.

[4] K. Iwasaki, T. Satoh-Nakagawa, M. Maruyama et al., "A randomized, observer-blind, controlled trial of the traditional Chinese medicine Yi-Gan San for improvement of behavioral and psychological symptoms and activities of daily living in dementia patients," Journal of Clinical Psychiatry, vol. 66, no. 2, pp. 248-252, 2005.
[5] K. Mizukami, T. Asada, T. Kinoshita et al., "A randomized cross-over study of a traditional Japanese medicine (kampo), yokukansan, in the treatment of the behavioural and psychological symptoms of dementia," International Journal of Neuropsychopharmacology, vol. 12, no. 2, pp. 191-199, 2009.

[6] K. Iwasaki, S. Kobayashi, Y. Chimura et al., "A randomized, double-blind, placebo-controlled clinical trial of the Chinese herbal medicine "ba wei di huang wan" in the treatment of dementia," Journal of the American Geriatrics Society, vol. 52, no. 9, pp. 1518-1521, 2004.

[7] Y. Shimada, K. Terasawa, T. Yamamoto et al., "A well-controlled study of Choto-san and placebo in the treatment of vascular dementia," Journal of Traditional Medicines, vol. 11, pp. 246-255, 1994.

[8] K. Terasawa, Y. Shimada, T. Kita et al., "Choto-san in the treatment of vascular dementia: a double-blind, placebo-controlled study," Phytomedicine, vol. 4, no. 1, pp. 15-22, 1997.

[9] M. Maruyama, N. Tomita, K. Iwasaki et al., "Benefits of combining donepezil plus traditional Japanese herbal medicine on cognition and brain perfusion in Alzheimer's disease: a 12week observer-blind, donepezil monotherapy controlled trial," Journal of the American Geriatrics Society, vol. 54, no. 5, pp. 869-871, 2006.

[10] M. Fujiwara and K. Iwasaki, "Toki-Shakuyaku-San and OrenGedoku-To improve the disruption of spatial cognition induced by cerebral ischaemia and central cholinergic dysfunction in rats," Phytotherapy Research, vol. 7, no. 7, pp. S60-S62, 1993.

[11] T. Itoh, S. Michijiri, S. Murai et al., "Regulatory effect of danggui-shaoyao-san on central cholinergic nervous system dysfunction in mice," American Journal of Chinese Medicine, vol. 24, no. 3-4, pp. 205-217, 1996.

[12] N. Hagino, "Kampo medicine and neuroendocrine-from here to molecular biology," Journal of Traditional Medicines, vol. 13, pp. 105-117, 1996.

[13] K. Toriizuka, P. Hou, T. Yabe, K. Iijima, T. Hanawa, and J. C. Cyong, "Effects of Kampo medicine, Toki-shakuyakusan (Tang-Kuei-Shao-Yao-San), on choline acetyltransferase 
activity and norepinephrine contents in brain regions, and mitogenic activity of splenic lymphocytes in ovariectomized mice," Journal of Ethnopharmacology, vol. 71, no. 1-2, pp. 133 143, 2000.

[14] I. Hatip-Al-Khatib, F. B. Hatip, Y. Yoshimitsu et al., "Effect of Toki-Shakuyaku-San on acetylcholine level and blood flow in dorsal hippocampus of intact and twice-repeated ischemic rats," Phytotherapy Research, vol. 21, no. 3, pp. 291-294, 2007.

[15] Q. H. Song, K. Toriizuka, G. B. Jin, T. Yabe, and J. C. Cyong, "Long term effects of Toki-shakuyaku-san on brain dopamine and nerve growth factor in olfactory-bulb-lesioned mice," Japanese Journal of Pharmacology, vol. 86, no. 2, pp. 183-188, 2001.

[16] N. Egashira, K. Iwasaki, Y. Akiyoshi et al., "Protective effect of Toki-shakuyaku-san on amyloid $\beta 25-35$ - induced neuronal damage in cultured rat cortical neurons," Phytotherapy Research, vol. 19, no. 5, pp. 450-453, 2005.

[17] Y. Y. Liu and T. Kojima, "Protective effects of Toki-ShakuyakuSan Tsumra Japan-23 (TJ-23) on $\beta$-amyloid protein $(\beta 40)$ induced apoptosis in pheochromocytoma-12 (PC12) cells," Journal of Health Science, vol. 53, no. 2, pp. 196-201, 2007.

[18] Y. Ueda, M. Komatsu, and M. Hiramatsu, "Free radical scavenging activity of the Japanese herbal medicine TokiShakuyaku-San (TJ-23) and its effect on superoxide dismutase activity, lipid peroxides, glutamate, and monoamine metabolites in aged rat brain," Neurochemical Research, vol. 21, no. 8, pp. 909-914, 1996.

[19] M. Ito, M. Ohbayashi, M. Furukawa, and S. Okoyama, "Neuroprotective effects of TJ-23 (Tokishakuyakusan) on adult rat motoneurons following peripheral facial nerve axotomy," Otolaryngology-Head and Neck Surgery, vol. 136, no. 2, pp. 225230, 2007.

[20] K. Inanaga, K. Dainoson, Y. Ninomiya et al., "Effects of TokiShakuyaku-San in patients with senile cognitive disorders," Progress in Medicine, vol. 16, pp. 293-300, 1996 (Japanese).

[21] Y. Kitabayashi, K. Shibata, T. Nakamae, J. Narumoto, and K. Fukui, "Effect of traditional Japanese herbal medicine tokishakuyaku-san for mild cognitive impairment: SPECT study," Psychiatry and Clinical Neurosciences, vol. 61, no. 4, pp. 447448, 2007.

[22] H. Goto, N. Satoh, Y. Hayashi et al., "A Chinese herbal medicine, tokishakuyakusan, reduces the worsening of impairments and independence after stroke: a 1-year randomized, controlled trial," Evidence-based Complementary and Alternative Medicine, vol. 2011, article 194046, 2011.

[23] R. C. Petersen, G. E. Smith, S. C. Waring, R. J. Ivnik, E. G. Tangalos, and E. Kokmen, "Mild cognitive impairment: clinical characterization and outcome," Archives of Neurology, vol. 56, no. 3, pp. 303-308, 1999.

[24] G. McKhann, D. Drachman, and M. Folstein, "Clinical diagnosis of Alzheimer's disease: report of the NINCDS-ADRDA work group under the auspices of Department of Health and Human Services Task Force on Alzheimer's disease," Neuro$\log y$, vol. 34, no. 7, pp. 939-944, 1984.

[25] M. F. Folstein, S. E. Folstein, and P. R. McHugh, “"Mini mental state". A practical method for grading the cognitive state of patients for the clinician," Journal of Psychiatric Research, vol. 12, no. 3, pp. 189-198, 1975.

[26] J. L. Cummings, M. Mega, K. Gray, S. Rosenberg-Thompson, D. A. Carusi, and J. Gornbein, "The neuropsychiatric inventory: comprehensive assessment of psychopathology in dementia," Neurology, vol. 44, no. 12, pp. 2308-2314, 1994.
[27] M. P. Lawton and E. M. Brody, "Assessment of older people: self-maintaining and instrumental activities of daily living," Gerontologist, vol. 9, no. 3, pp. 179-186, 1969.

[28] B. A. Vogt and S. Laureys, "Posterior cingulate, precuneal and retrosplenial cortices: cytology and components of the neu ral network correlates of consciousness," Progress in Brain Research, vol. 150, pp. 205-217, 2005.

[29] B. A. Vogt, D. M. Finch, and C. R. Olson, "Functional heterogeneity in cingulate cortex: the anterior executive and posterior evaluative regions," Cerebral Cortex, vol. 2, no. 6, pp. 435-443, 1992.

[30] Y. Zhou, J. H. Dougherty Jr., K. F. Hubner, B. Bai, R. L. Cannon, and R. K. Hutson, "Abnormal connectivity in the posterior cingulate and hippocampus in early Alzheimer's disease and mild cognitive impairment," Alzheimer's and Dementia, vol. 4, no. 4, pp. 265-270, 2008.

[31] R. Heun, K. Freymann, M. Erb et al., "Successful verbal retrieval in elderly subjects is related to concurrent hippocampal and posterior cingulate activation," Dementia and Geriatric Cognitive Disorders, vol. 22, no. 2, pp. 165-172, 2006.

[32] M. Alegret, G. Vinyes-Junqué, M. Boada et al., "Brain perfusion correlates of visuoperceptual deficits in mild cognitive impairment and mild Alzheimer's disease," Journal of Alzheimer's Disease, vol. 21, no. 2, pp. 557-567, 2010.

[33] N. Hirono, E. Mori, K. Ishii et al., "Hypofunction in the posterior cingulate gyrus correlates with disorientation for time and place in Alzheimer's disease," Journal of Neurology Neurosurgery and Psychiatry, vol. 64, no. 4, pp. 552-554, 1998.

[34] R. T. Staff, H. G. Gemmell, M. F. Shanks, A. D. Murray, and A. Venneri, "Changes in the rCBF images of patients with Alzheimer's disease receiving Donepezil therapy," Nuclear Medicine Communications, vol. 21, no. 1, pp. 37-41, 2000.

[35] S. Nakano, T. Asada, H. Matsuda, M. Uno, and M. Takasaki, "Donepezil hydrochloride preserves regional cerebral blood flow in patients with Alzheimer's disease," Journal of Nuclear Medicine, vol. 42, no. 10, pp. 1441-1445, 2001.

[36] Y. Ushijima, C. Okuyama, S. Mori, T. Kubota, T. Nakai, and T. Nishimura, "Regional cerebral blood flow in Alzheimer's disease: comparison between short and long-term donepezil therapy," Annals of Nuclear Medicine, vol. 20, no. 6, pp. 425429, 2006.

[37] S. Shimizu, H. Hanyu, T. Iwamoto, K. Koizumi, and K. Abe, "SPECT follow-up study of cerebral blood flow changes during Donepezil therapy in patients with Alzheimer's disease," Journal of Neuroimaging, vol. 16, no. 1, pp. 16-23, 2006. 


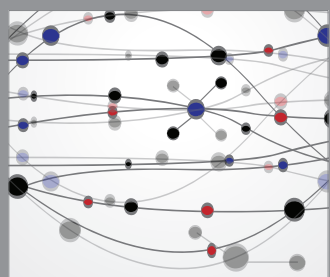

The Scientific World Journal
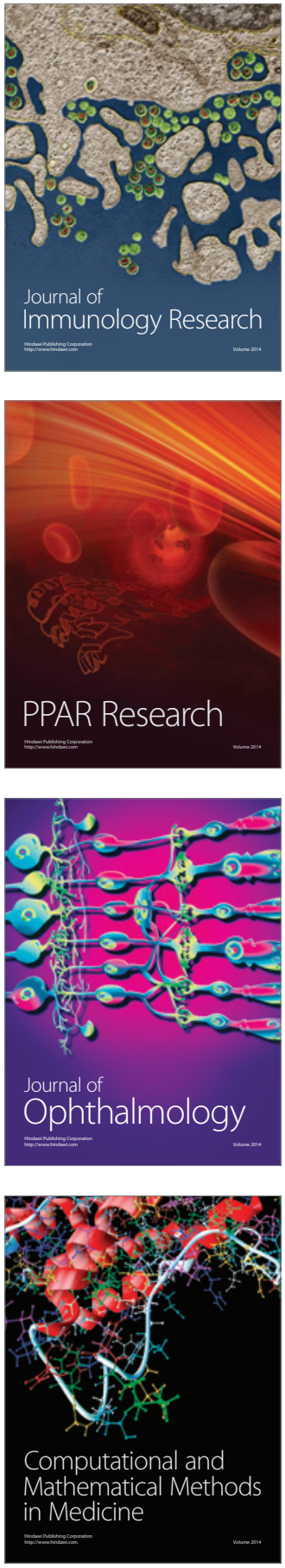

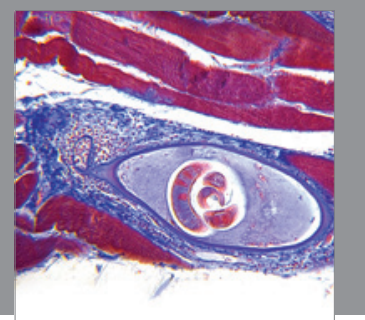

Gastroenterology

Research and Practice
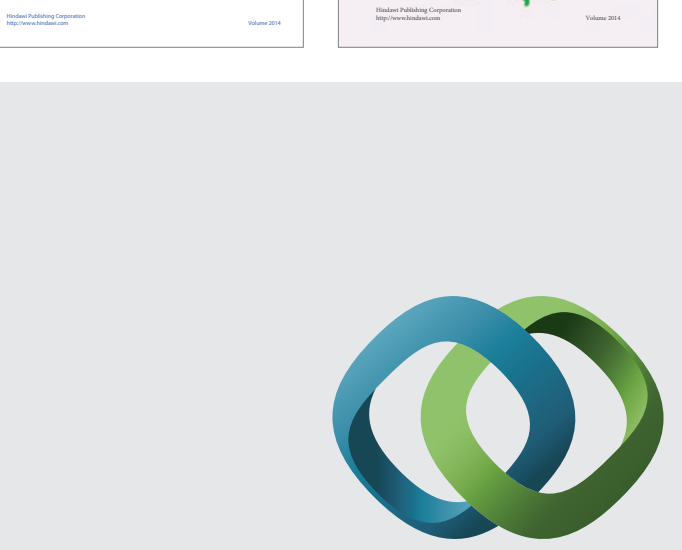

\section{Hindawi}

Submit your manuscripts at

http://www.hindawi.com
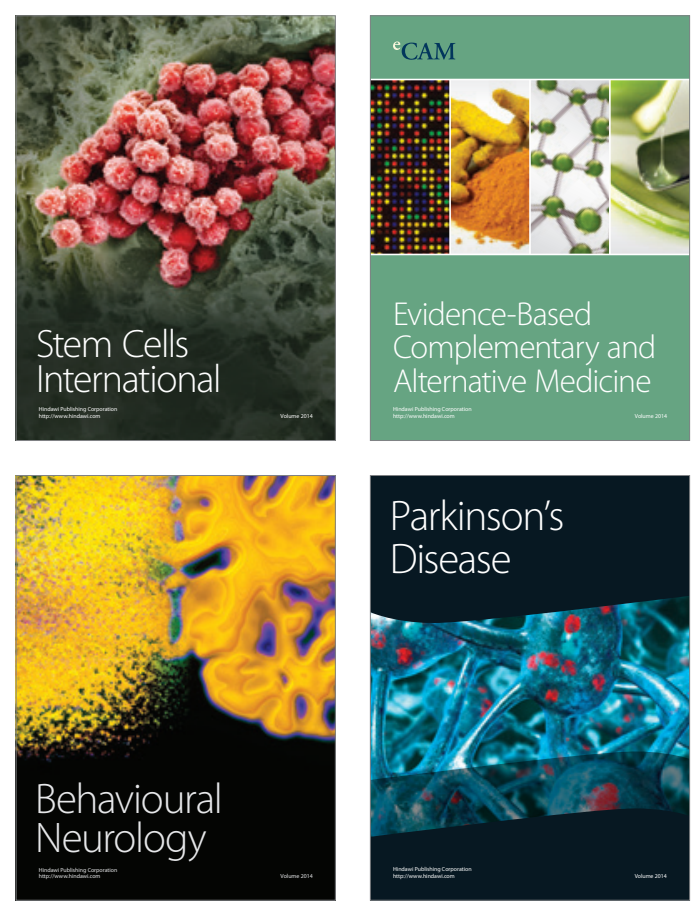

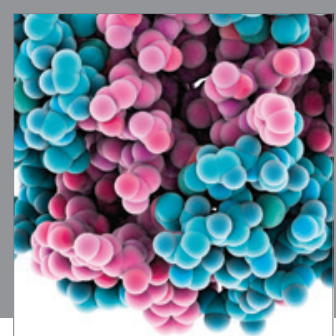

Journal of
Diabetes Research

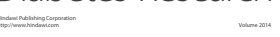

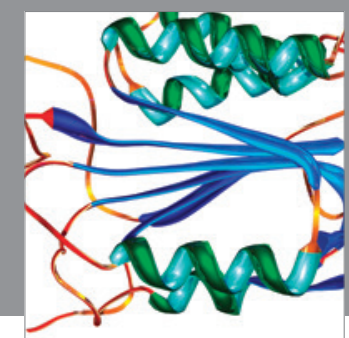

Disease Markers
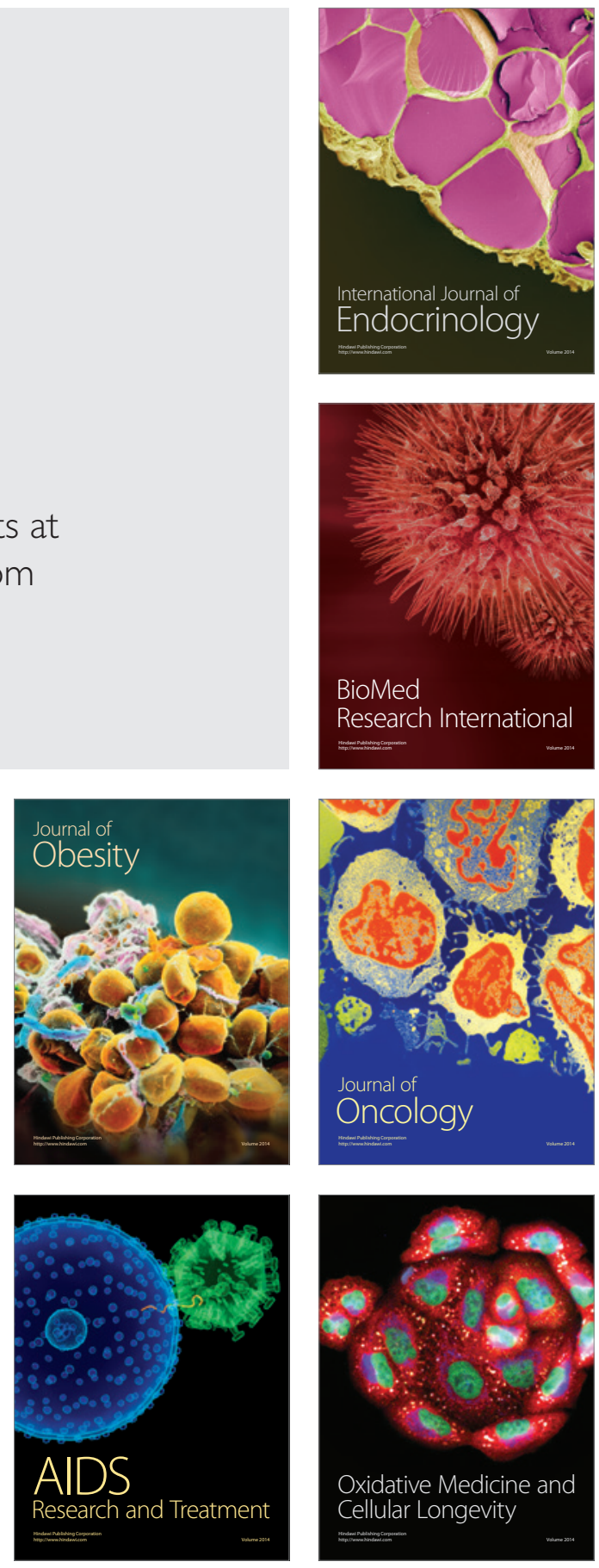